\title{
Ensayos de resistencia a la compresión de bloques de suelo cemento
}

\section{Test of resistance to compression of soil-cement blocks}

\author{
J.M. Mas ${ }^{(* * *)}$, C. Kirschbaum ${ }^{(* *+)}$
}

RESUMEN

El objetivo de este trabajo es determinar la dosificación más adecuada para un determinado tipo de suelo, que se utilizará para fabricar bloques de suelo-cemento, para construir una vivienda en El Puestito, Burruyacu, Tucumán, Argentina.

Se extrajeron dos muestras de suelo del área estudiada y se fabricaron probetas de suelo-cemento, utilizando la mezcla de dos muestras a la vez y con cada una por separado. Otra variable introducida fue la presión de fabricación. A los 28 días, fueron ensayadas de dos maneras: la primera, sometiéndolas a la compresión hasta la rotura y la segunda, sumergiéndolas en agua, pesándolas en intervalos de tiempos determinados.

Los resultados mostraron que: aumentando la presión de fabricación, se aumenta la resistencia a la compresión, mientras que la absorción disminuye. Las dosificaciones con mayor cantidad de suelo 1 con respecto a la de suelo 2, producen un aumento de la resistencia a la rotura.
SUMMARY

The purpose of this study is to determine the proper dosage for a particular type of soil, which will be used to make soil-cement blocks to build a home in El Puestito, Burruyacu, Tucumán, Argentina.

Two soil samples were extracted from studied area and samples of soil-cement specimens, using a mixture of two samples simultaneously and with each one separately. Another variable introduced was the pressure of production. At 28 days, were tested in two ways: first, subjecting them to compression at break and the second tests was made by submerging them in water and weighing them at certain time intervals.

The results showed that: increasing the pressure of production, increases resistance to compression, while the absorption decreases. The results also showed that greater amounts of soil 1, enhances the resistance to breakage.

Keywords: Soil-cement, rural housing, blocks, dosage, absorption.
Palabras clave: suelo-cemento, vivienda rural, bloques, dosificación, absorción. 
1. Plano de ubicación del área en estudio.

2. Prototipo de vivienda diseñado para la localidad rural "El Puestito".

\section{INTRODUCCION}

En el marco de un proyecto de investigación sobre desarrollo de poblaciones rurales, PICTO 2004 N 870 "Tecnologías para el hábitat, el aprovechamiento energético y el desarrollo productivo en áreas rurales de Tucumán", se diseñó un prototipo de vivienda para familias minifundistas. El área de estudio y análisis es la comuna rural El Puestito $\left(26^{\circ} 24\right.$ > 0 » Sud, $64^{\circ} 47$ > 0» Oeste), ubicada en el departamento de Burruyacu, a $75 \mathrm{Km}$ al noreste de la ciudad de San Miguel de Tucumán, capital de la provincia de Tucumán, Argentina. Ver Figura 1.
La comuna ocupa, en su mayor parte, el piedemonte de las Sierras de Medina zona de la selva húmeda denominada Las Yungas. Hacia el este se vincula con la llanura chaqueña. El clima es cálido con lluvias principalmente en el verano. Las precipitaciones varían entre $400-600 \mathrm{~mm}$ por año.

La zona es apta para una variada actividad agropecuaria compuesta por cultivos tanto tradicionales como caña de azúcar, maíz, trigo, citrus, palta y los introducidos en los últimos años con importante crecimiento de la soja y la cría de vacunos, cerdos y caballos.

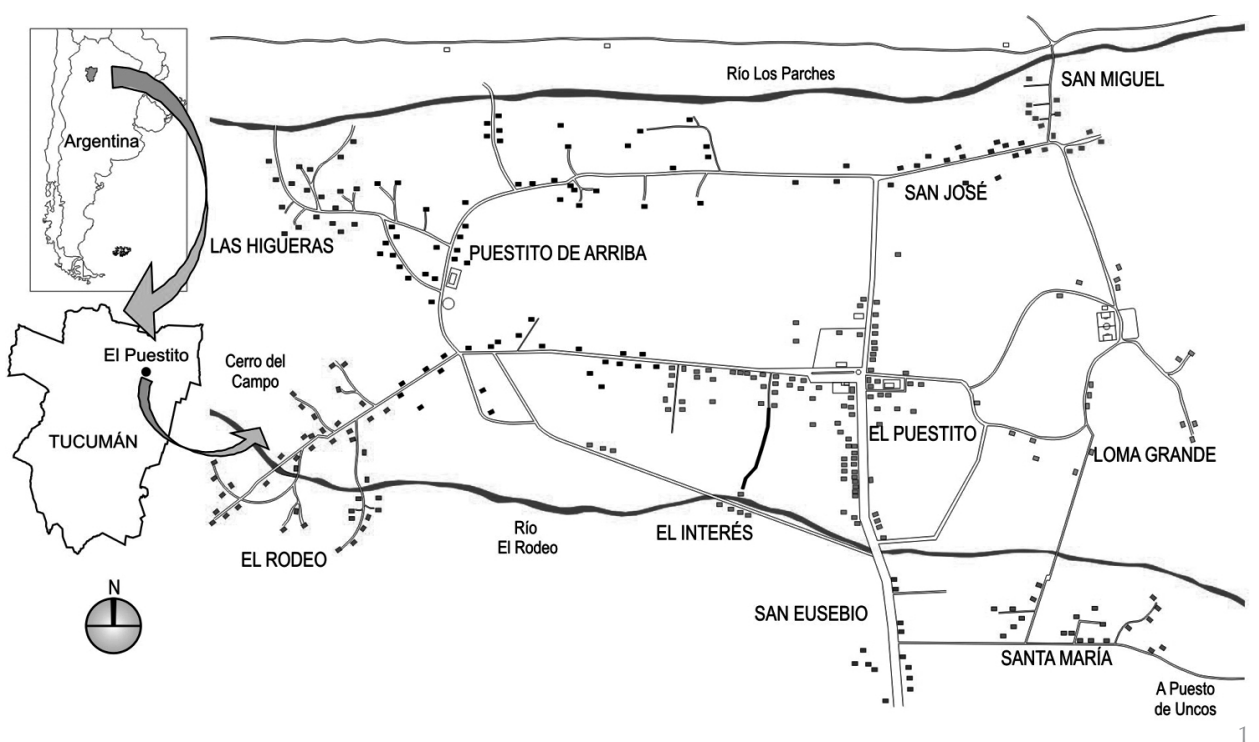

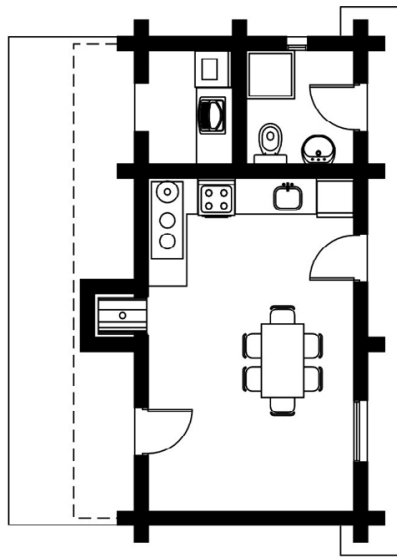
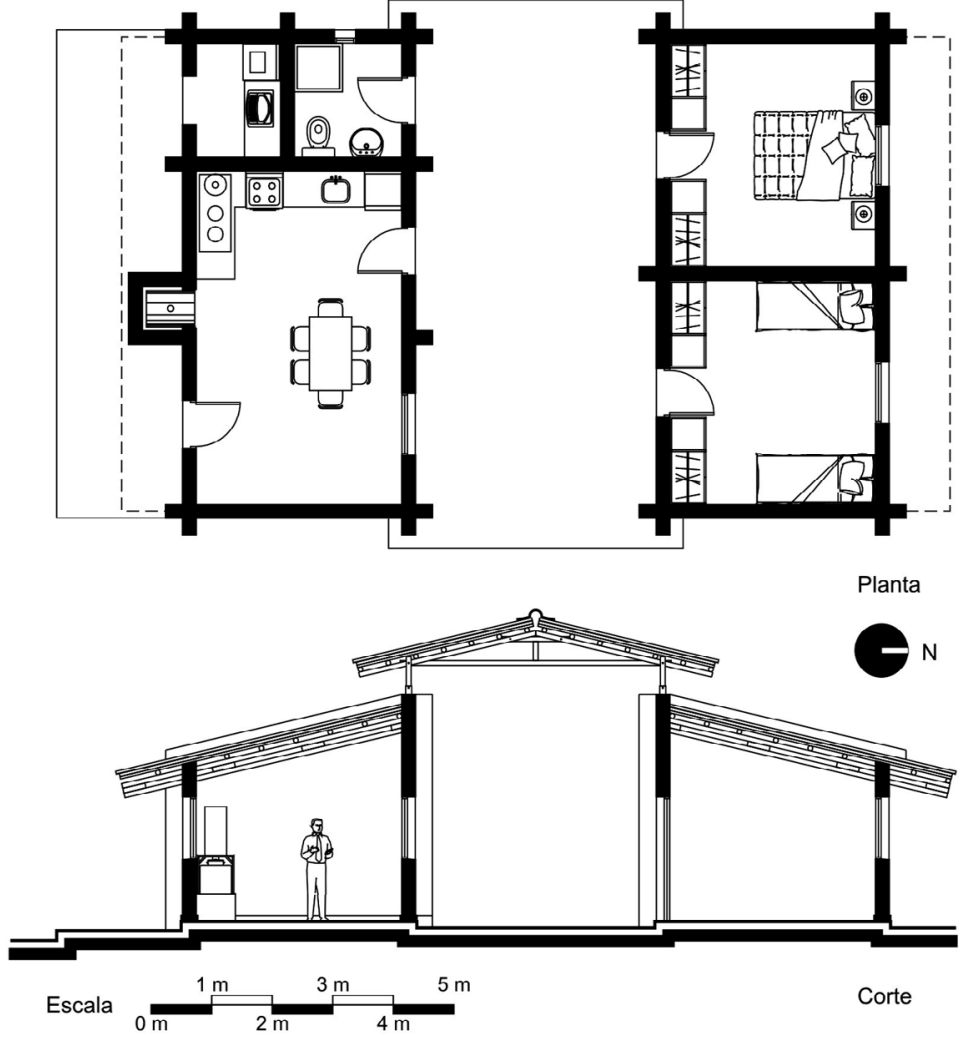

Es zona propicia para las actividades de granja como el cultivo de hortalizas y la cría de aves de corral, tales como gallinas y pavos, que en gran proporción están orientadas a atender la subsistencia familiar.

El lugar posee un importante patrimonio en flora y fauna natural en cerros, valles y bosques. La existencia de terrenos quebrados y la presencia de pequeños propietarios en la mayor parte del territorio, distribuidos en pequeños y medianos predios de entre $0,5 \mathrm{a}$ 50 hectáreas, actúan también como barrera para la explotación extensiva de los suelos y recursos naturales.

El trabajo constó de varias etapas. En la primera se realizó un diagnóstico general del área, considerando la relación de los habitantes con el medio ambiente y el paisaje, efectos de las condiciones de las viviendas en la salud y bienestar de los ocupantes, las características de ellas, necesidades, demandas y deseos de los habitantes del lugar, analizando las características socioculturales que hacen a la apropiación del espacio y de la vivienda por parte de los moradores, es decir a su modo de vivir $(1,2)$. 
Una vez realizado el diagnóstico, se enunciaron las pautas a seguir para satisfacer las demandas de los pobladores. Considerando las características climáticas del lugar y los materiales disponibles en la zona, se procedió a diseñar un prototipo de vivienda. El mismo consta de dos módulos rectangulares unidos por un espacio semicubierto que los vincula (3). Ver Figura 2.

Actualmente se encuentra en construcción en el área estudiada, un modelo experimental del prototipo de vivienda diseñado. El trabajo se realiza con la colaboración de pobladores y de personal que trabaja en la comuna. Ver Figura 3.

El presente artículo trata específicamente la manera en que se determinó la dosificación más adecuada para la fabricación de bloques comprimidos de suelo-cemento, que serán utilizados para la materialización de la envolvente vertical.

\section{LA ENVOLVENTE}

La envolvente de un edificio tiene, entre otras, las funciones de actuar como elemento moderador de las condiciones ambientales externas y delimitar los espacios arquitectónicos en donde se desarrollan las distintas actividades humanas, con el fin de lograr un ambiente interior confortable, eficiente y saludable, en otras palabras, estar preparada para brindar a sus ocupantes la protección contra las agresiones externas: Iluvia, ruido, radicación solar, por nombrar solo algunas.

Está compuesta por cerramientos horizontales y verticales. Entre los horizontales encontramos al techo y al piso. Los verticales se refieren a los muros y aberturas. Los primeros pueden ser realizados con diferentes materiales y métodos constructivos, como por ejemplo, mampuestos de ladrillos cerámicos comunes, bloques de hormigón, piedra, etc. Se conocen como mampuestos, a aquellos que son colocados en forma manual y superpuestos. Así se tiene que:

Las mamposterías en general constituyen sistemas constructivos realizados mediante técnicas de ejecución simples. Estas se basan en la superposición ordenada de mampuestos unidos con mezclas de asiento, formando juntas horizontales y verticales, con el objeto de obtener un comportamiento monolítico frente a la acción de cargas exteriores (4).

Cada uno de los elementos de la envolvente debe estar diseñado para soportar los esfuerzos o solicitaciones a las que están sometidos. Mientras que, por ejemplo, una losa está sometida a esfuerzos de flexión y

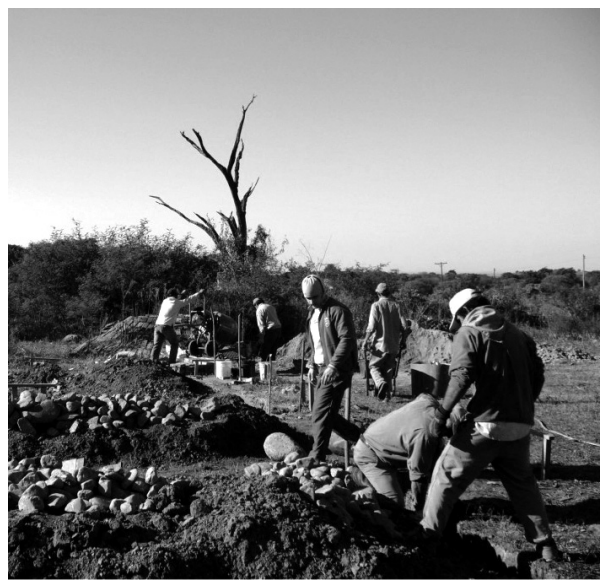

3. Construcción del prototipo en el área estudiada. corte, un muro generalmente trabaja a la compresión. Por ello es de suma importancia que los mampuestos con los que se pretende materializar la envolvente vertical, en nuestro caso en particular, bloques de suelo-cemento, deban tener una adecuada resistencia a esta solicitación, de tal manera que les permita soportar convenientemente los esfuerzos a los que se vean sometidos.

Los bloques de suelo-cemento comprimidos, tienen la ventaja frente a otros conformados por materiales industrializados como son los ladrillos cerámicos comunes o huecos, de poder ser diseñados para responder a las necesidades de cada caso en particular $(5,6)$. Por ello el diseño de un bloque de suelo-cemento comprende la cuantificación o dosificación de la cantidad necesaria de cada uno de los componentes que forman la mezcla a emplear en la fabricación. Las variaciones en las cantidades a utilizar de cemento, tierra y agua, modifican las propiedades del elemento.

Estos bloques, además de ser de bajo costo, tienen otra ventaja interesante de destacar que es el uso de la tierra que, al mismo tiempo de ser una material fácil de obtener, tiene un impacto ecológico muy importante ya que, por ejemplo, para la cocción de ladrillos cerámicos comunes se quema leña, lo que provoca daños al medioambiente. Además es importante destacar que en la construcción, el costo del flete es alto. Usando la tierra del lugar donde se planea construir se ahorra dinero.

\section{METODOLOGÍA}

Como primera instancia se obtuvo, según indicaciones de la administración de la comuna, de los lugares de donde era posible extraer tierra, dos muestras de suelo de sectores diferentes del área de estudio. Las mismas fueron sometidas a ensayos, llevados a cado en el Laboratorio de suelos de la Facultad de Ciencias Exactas y Tecnología de la Univer- 
Tabla 1

Límite líquido, límite plástico e índice de plasticidad de las muestras de suelo extraídas

\begin{tabular}{|l|c|c|}
\hline & \multicolumn{2}{|c|}{ SUELO } \\
\hline & Muestra $\mathbf{N}^{\mathbf{0}}$ & Muestra $\mathbf{N}^{\circ} \mathbf{2}$ \\
\hline Límite líquido & 38,8 & No plástico \\
\hline Límite plástico & 21,0 & No plástico \\
\hline Índice de plasticidad & 17,8 & No plástico \\
\hline
\end{tabular}

sidad Nacional de Tucumán, con el fin de determinar su límite plástico, límite líquido y su índice de plasticidad. En Tabla 1 pueden verse los resultados obtenidos.

Una vez conocidas las características de los suelos, se fabricó probetas de suelo-cemento, haciendo dosificaciones con la mezcla de las dos muestras a la vez y con cada una por separado, mezclando los materiales en volúmenes aparentes en estado seco, adicionando la cantidad de agua necesaria para obtener una mezcla de bajo tenor de humedad y de aspecto casi seco.

Luego de elaboradas las mezclas, se llenaron probetas cilíndricas de $6 \mathrm{~cm}$ de diámetro y se las sometió a dos presiones distintas de fabricación: $5 \mathrm{Kg} / \mathrm{cm}^{2}$ y $10 \mathrm{Kg} / \mathrm{cm}^{2}$. Para cada una de las variables utilizadas se realizaron 6 probetas.

A los 28 días, y luego del proceso de secado, 5 de cada grupo, fueron sometidas a la compresión con una prensa manual, reservando la restante de cada serie para realizar la prueba de absorción. La misma se llevó a cabo sumergiéndolas completamente en agua a temperatura ambiente, tomando la lectura de su peso en el tiempo establecido por norma IRAM No 12588 (7). La Tabla 2 resume lo anteriormente expuesto.

\section{RESULTADOS}

\subsection{Resistencia a la compresión}

Los datos obtenidos muestran que el aumento de la resistencia a la compresión responde a dos variables, por un lado al aumento de la presión de fabricación y por otro al aumento de la proporción de tierra de la muestra 1 con respecto a la de la muestra 2. Así se tiene que con una dosificación igual a 1:8:2 y a $5 \mathrm{Kg} / \mathrm{cm}^{2}$ como presión de fabricación, se obtiene una resistencia a la rotura de $41,9 \mathrm{Kg} /$ $\mathrm{cm}^{2}$. Al mantener la misma dosificación y aumentar la presión de fabricación hasta los $10 \mathrm{Kg} / \mathrm{cm}^{2}$ existe un incremento de resistencia a la rotura de alrededor del $15,9 \%$ con respecto al valor anterior, es decir que se eleva hasta los 48,6 Kg/cm².

Al variar la dosificación y utilizar una igual a 1:7:3, se mantuvo la misma tendencia, es decir hubo un incremento de la resistencia a la rotura conforme aumenta la presión de fabricación. Para $5 \mathrm{Kg} / \mathrm{cm}^{2}$ de presión de fabricación, el valor de la resistencia a la rotura fue de $32,6 \mathrm{Kg} / \mathrm{cm}^{2}$, mientras que para $10 \mathrm{Kg} / \mathrm{cm}^{2}, 34,8 \mathrm{Kg} /$ $\mathrm{cm}^{2}$, lo que muestra un incremento del $6,7 \%$ la resistencia de la segunda contra la primera.

Tabla 2

Cantidad de probetas realizadas según distintas dosificaciones y presiones de fabricación

\begin{tabular}{|c|c|c|c|c|c|c|}
\hline \multicolumn{3}{|c|}{ Dosificación } & \multirow{2}{*}{$\begin{array}{c}\text { Presión de } \\
\text { fabricación }\end{array}$} & \multicolumn{3}{c|}{ Cantidad de probetas } \\
\cline { 1 - 1 } Cemento & Muestra 1 & Muestra 2 & \multirow{2}{*}{$\left(\right.$ Kg/cm $\left.{ }^{2}\right)$} & Fabricadas & $\begin{array}{c}\text { P/ensayo de } \\
\text { Compresión }\end{array}$ & $\begin{array}{c}\text { P/ensayo de } \\
\text { Absorción }\end{array}$ \\
\hline 1 & 8 & 2 & 5 & 6 & 5 & 1 \\
\hline 1 & 7 & 3 & 5 & 6 & 5 & 1 \\
\hline 1 & 6 & 4 & 5 & 6 & 5 & 1 \\
\hline 1 & 10 & $/$ & 5 & 6 & 5 & 1 \\
\hline 1 & 8 & $/$ & 5 & 6 & 5 & 1 \\
\hline 1 & $/$ & 10 & 5 & 6 & 5 & 1 \\
\hline 1 & $/$ & 8 & 5 & 6 & 5 & 1 \\
\hline 1 & 8 & 2 & 10 & 6 & 5 & 1 \\
\hline 1 & 7 & 3 & 10 & 6 & 5 & 1 \\
\hline 1 & 6 & 4 & 10 & 6 & 5 & 1 \\
\hline 1 & 10 & $/$ & 10 & 6 & 5 & 1 \\
\hline 1 & 8 & $/$ & 10 & 6 & 5 & 1 \\
\hline 1 & $/$ & 10 & 10 & 6 & 5 & 1 \\
\hline 1 & $/$ & 8 & 10 & 6 & 5 & 1 \\
\hline
\end{tabular}


Al utilizar una mezcla de dosificación igual a $1: 6: 4$, manteniendo constantes las mismas presiones de fabricación, se obtuvieron resistencias a la rotura con valores de $28,3 \mathrm{Kg} / \mathrm{cm}^{2}$ y $32,7 \mathrm{Kg} / \mathrm{cm}^{2}$, lo que representa un incremento de $15,5 \%$.

Los mejores resultados se obtuvieron con la muestras de suelo número 1 , ya que la resistencia a la compresión siempre es mayor en las probetas fabricadas con ella. Así se tiene que para una dosificación 1:10 de la muestra 1 , se obtienen, a $5 \mathrm{Kg} / \mathrm{cm}^{2}$ de presión de fabricación, $59,5 \mathrm{Kg} / \mathrm{cm}^{2}$, mientras que la misma dosificación y presión de fabricación pero con muestra del suelo tipo 2, nos da $32,8 \mathrm{Kg} / \mathrm{cm}^{2}$ de resistencia. En el caso de utilizar una dosificación de 1:8, se obtiene $75,4 \mathrm{Kg} / \mathrm{cm}^{2}$ para el suelo 1 y $39,8 \mathrm{Kg} / \mathrm{cm}^{2}$ para el suelo 2.

Cabe aclarar que, si se mantienen las dosificaciones y se aumenta la presión de fabricación, aumenta también la resistencia a la rotura. Es así que una dosificación de 1:10 a $10 \mathrm{Kg} / \mathrm{cm}^{2}$, nos da una resistencia de $64,9 \mathrm{Kg} / \mathrm{cm}^{2}$ para el suelo 1 y de $32,4 \mathrm{Kg} / \mathrm{cm}^{2}$ para el 2 . Por último, con una mezcla de $1: 8$ a $10 \mathrm{Kg} / \mathrm{cm}^{2}$, se obtuvo, para el suelo 1 , un valor de $85,1 \mathrm{Kg} / \mathrm{cm}^{2}$ y para el suelo 2 , de $42,5 \mathrm{Kg} / \mathrm{cm}^{2}$.

Como era de esperar y como puede entenderse por los resultados obtenidos, a medida que aumenta la proporción de cemento con respecto a la tierra, aumenta también la resistencia a la compresión, sin importar la muestra utilizada ni la presión de fabricación.

Las Tablas 3 y 4 resumen lo antedicho.

\subsection{Absorción}

Debido a la absorción de agua, todas las probetas, sin importar la mezcla con las que fueron realizadas ni la presión a la que fueron sometidas durante la fabricación, registraron un aumento de su peso, dependiendo la cantidad de agua absorbida del tipo de mezcla y la presión de fabricación. Es así que se obtuvo como valor máximo, a las 24 horas de sumergida la probeta, un incremento del $21,9 \%$ del peso de la probeta en estado seco, mientras que el valor mínimo, registrado al minuto fue de $1,0 \%$.

La tendencia general indica que a mayor presión de fabricación se obtiene un menor porcentaje de absorción de humedad, sin importar la dosificación que se utilice. Esto puede explicarse por el hecho de que al someter a una mezcla durante la fabricación a una presión mayor, se obtiene una probeta con mayor grado de compacidad, es decir con menor porcentaje de vacíos, por lo tanto con menor capacidad de absorber agua.
Así se tiene que las probetas fabricadas con la mezcla A disminuyen su absorción en un 9\% aproximadamente, cuando su presión de fabricación varía de $5 \mathrm{Kg} / \mathrm{cm}^{2}$ a $10 \mathrm{Kg} / \mathrm{cm}^{2}$. Para el caso de la mezcla $\mathrm{B}$, los resultados muestran la misma tendencia ya que la absor-

Tabla 3

Resistencia promedio a la rotura, según dosificación y presión de fabricación para mezclas realizadas con combinación de las dos muestras a la vez.

\begin{tabular}{|c|c|c|c|c|c|c|c|c|c|c|}
\hline \multirow{2}{*}{ ID } & \multicolumn{3}{|c|}{ Dosificación } & \multirow{2}{*}{$\begin{array}{c}\text { Presión de } \\
\text { fabricación } \\
\text { (Kg) }\end{array}$} & \multirow{2}{*}{$\begin{array}{l}\mathrm{T}\left(\mathrm{Kg} / \mathrm{cm}^{2}\right) \\
\text { Promedio }\end{array}$} & \multicolumn{5}{|c|}{ Probetas } \\
\hline & Cto & M1 & M2 & & & 1 & 2 & 3 & 4 & 5 \\
\hline A & 1 & 8 & 2 & 5,0 & 41,9 & 41,2 & 38,5 & 42,5 & 43,0 & 44,5 \\
\hline B & 1 & 7 & 3 & 5,0 & 32,6 & 30,7 & 32,0 & 33,0 & 33,2 & 34,0 \\
\hline $\mathrm{C}$ & 1 & 6 & 4 & 5,0 & 28,3 & 27,5 & 27,2 & 28,0 & 28,7 & 30,2 \\
\hline $\mathrm{D}$ & 1 & 8 & 2 & 10,0 & 48,6 & 47,5 & 48,0 & 49,0 & 48,2 & 50,2 \\
\hline $\mathrm{E}$ & 1 & 7 & 3 & 10,0 & 34,8 & 34,7 & 35,5 & 35,0 & 34,2 & 34,7 \\
\hline $\mathrm{F}$ & 1 & 6 & 4 & 10,0 & 32,7 & 32,5 & 31,7 & 31,2 & 33,5 & 34,5 \\
\hline
\end{tabular}

Tabla 4

Resistencia promedio a la rotura, según dosificación y presión de fabricación para mezclas de una sola muestra por vez.

\begin{tabular}{|c|c|c|c|c|c|c|c|c|c|c|}
\hline \multirow{2}{*}{ ID } & \multicolumn{3}{|c|}{ Dosificación } & \multirow{2}{*}{$\begin{array}{c}\text { Presión de } \\
\text { fabricación } \\
\text { (Kg) }\end{array}$} & \multirow{2}{*}{$\begin{array}{l}\mathrm{T}\left(\mathrm{Kg} / \mathrm{cm}^{2}\right) \\
\text { Promedio }\end{array}$} & \multicolumn{5}{|c|}{ Probetas } \\
\hline & Cto & M1 & M2 & & & 1 & 2 & 3 & 4 & 5 \\
\hline G & 1 & 10 & / & 5,0 & 59,5 & 60,0 & 61,2 & 57,5 & 57,5 & 61,2 \\
\hline $\mathrm{H}$ & 1 & / & 10 & 5,0 & 32,8 & 28,7 & 30,5 & 32,0 & 38,5 & 34,5 \\
\hline I & 1 & 8 & I & 5,0 & 75,4 & 71,2 & 78,7 & 74,9 & 77,9 & 74,4 \\
\hline J & 1 & I & 8 & 5,0 & 39,8 & 36,2 & 39,0 & 40,0 & 41,2 & 42,5 \\
\hline K & 1 & 10 & I & 10,0 & 64,9 & 65,0 & 63,7 & 62,0 & 65,0 & 68,7 \\
\hline $\mathrm{L}$ & 1 & / & 10 & 10,0 & 32,4 & 31,2 & 32,7 & 33,7 & 30,5 & 34,0 \\
\hline$M$ & 1 & 8 & / & 10,0 & 85,1 & 86,4 & 91,2 & 81,2 & 74,4 & 92,4 \\
\hline $\mathrm{N}$ & 1 & / & 8 & 10,0 & 42,5 & 43,7 & 41,2 & 46,7 & 39,0 & 41,7 \\
\hline
\end{tabular}

ción también disminuye, en este caso en un $16 \%$ aproximadamente. Para la mezcla $C$ se observó una disminución del orden del 17\%.

Si analizamos la tendencia para pobretas fabricadas con suelos de una sola muestra por vez, se tienen una disminuciones aproximadas del $25 \%$ para la mezcla G, $20 \%$ para la $\mathrm{H}, 23 \%$ para la I y $19 \%$ para la J. Las letras que identifican a cada mezcla, corresponden al ID utilizado en Tabla 4.

\section{Ver Figuras 4 y 5.}

\section{CONCLUSIONES}

Los resultados obtenidos demuestran que:

- A medida que aumenta la presión a la que son sometidas las probetas de suelo-cemento durante el proceso de fabricación, aumenta también su resistencia a la compresión mientras que la absorción disminuye. 
4. Variación de la absorción en función del tiempo de inmersión para dosificaciones que utilizan mezclas de las dos muestras a la vez.

5. Variación de la absorción en función del tiempo de inmersión para mezclas que utilizan una sola muestra por vez.
- Las dosificaciones en las cuales se aumenta la relación entre la cantidad de suelo 1 y la de suelo 2 se produce un aumento de la resistencia a la rotura, sin importar la presión de fabricación.

Los mejores rendimientos se obtuvieron utilizando para la fabricación de las probetas, morteros realizados con la muestra de suelo 1. Se recomienda no utilizar para este caso particular mezclas de los dos suelos, ya que las resistencias obtenidas son menores que se si utiliza el suelo 1 solamente. necesidades de resistencia y economía es la mezcla K (ver Tabla 4), por lo que será utilizada para la fabricación de los bloques de suelo cemento que se utilizarán en la construcción del prototipo propuesto.

\section{RECONOCIMIENTOS}

Este trabajo se realiza en el marco del proyecto PICTO 2004 № 870 financiado por la Universidad Nacional de Tucumán y la Agencia Nacional de Promoción Científica y Tecnológica, Argentina.
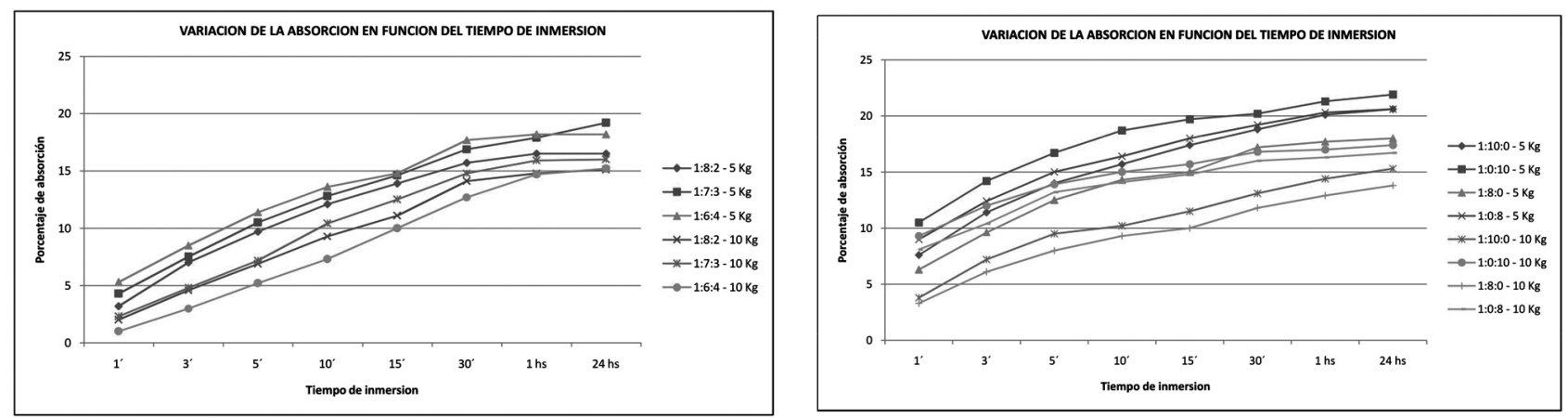

El suelo 2 utilizado por separado no da buenos resultados en cuanto a las resistencias a la compresión logradas. Otra desventaja es que en general absorben una mayor cantidad de agua, lo que disminuiría aun más su resistencia.

Si comparan los valores obtenidos en las diferentes mezclas realizadas, puede determinarse que, la que mejor se adapta a las
Las tareas de laboratorio se realizaron en las instalaciones del Centro Regional de Investigaciones de Arquitectura de Tierra Cruda (CRIATiC), de la Facultad de Arquitectura y Urbanismo de la Universidad Nacional de Tucumán. Un agradecimiento especial al Arq. Rafael Mellace (Director del CRIATiC) y al Ing. Carlos Alderete (Integrante del CRIATiC), por permitirnos usar sus instalaciones.

\section{BIBLIOGRAFÍA}

(1) Mas, J.: Análisis de Viviendas en un Área Rural de la Provincia de Tucumán. En:Terceras Jornadas de la Asociación Argentino Uruguaya de Economía Ecológica ASAUEE. Tucumán, Argentina. 2007, 10 p.S

(2) Tonello, G.; Mas, J.; Raitelli, M.; Kirschbaum, C.: Factores Ambientales en Viviendas de una Zona Rural y su Efecto en las Personas. En: V Congreso de Medio Ambiente. La Plata, Buenos Aires, Argentina, 2007, 15 p.

(3) Mas, J.: Prototipo de Vivienda para un Área Rural de la Provincia de Tucumán. En: Segundas Jornadas de Jóvenes Investigadores. Tucumán, Argentina. 2008, 15 p.

(4) Arias, L.; Alderete, C.; Mellace, R.: Variación de la Resistencia del BTC según distintos Estados

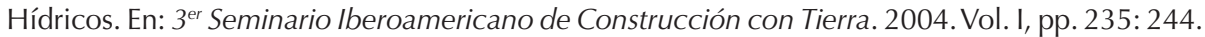

(5) Arias, L.; Alderete, C.; Gonzalo, G.: Comportamiento T-H de bloques comprimidos de suelo

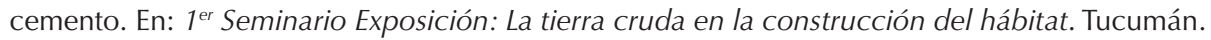
2002. 10 p.

(6) CECOVI: Tierra, Tecnología y Casa Propia. Revista de la Facultad Regional Santa Fe-UTN, n. ${ }^{0} 14$. Proyecto Aldeas Rurales Escolares (FUNDARE-UTN). [En-línea]. 2004. [Fecha de consulta: 15 de octubre de 2009]. Disponible en: http://cilad.com/guiasolidaria.pccp.net.ar/documentos/ vivienda-tierra.htm.

(7) IRAM 12588: Capacidad de absorción de agua por inmersión en agua fría. 1982, 10 p. 


\title{
Problemas asociados al equilibrio en estructuras de membrana con bordes rígidos y cables
}

\author{
Equilibrium problems in membrane structures with rigid and \\ cable boundaries \\ G. Viglialoro $^{(*)}$, J. Murcia ${ }^{(* *)}$
}

RESUMEN

Este trabajo aborda el análisis del equilibrio de una membrana con bordes rígidos y cables de borde para la fase de pretensado. La idea de utilizar las membranas en aplicaciones como las pasarelas, una nueva tecnología que está siendo desarrollada en España, implica niveles más altos de responsabilidad y de esfuerzos, requiriendo así un análisis estructural ajustado. La membrana y sus bordes se identifican, respectivamente, a una superficie con curvatura de Gauss negativa y a curvas cuya curvatura depende de las características estructurales de los bordes. El equilibrio se expresa mediante problemas de contorno con ecuaciones en derivadas parciales, en términos de la forma de la membrana y de su tensor de esfuerzos, así como de la forma y las cargas de los bordes. En particular, el equilibrio asociado a un cable de borde lleva a una condición de frontera muy singular que dificulta un tanto el análisis. A partir de ello, caben dos enfoques complementarios, llamados problema directo y problema dual. Se definen y analizan ambos problemas de contorno, estudiando sus principales aspectos cualitativos. Asimismo, con el objetivo de obtener resultados prácticos, se propone un procedimiento general de resolución numérica para el problema directo.

445-24

Palabras clave: Membrana; Pasarela; Borde rígido; Cable de borde; Problema directo; Problema dual.
SUMMARY

This paper presents the equilibrium analysis of a membrane with rigid and cable boundaries for the so called prestressing phase. The idea of using membranes in Civil Engineering applications such as footbridges, a new technology being developed in Spain, implies higher structural responsibility and more accurate analysis procedure. The membrane and its boundary are respectively identified to a regular and negative gaussian curvature surface and a set of regular curves whose curvature depends on the structural elements, rigid or cable. Equilibrium is directly expressed by means of boundary differential problems, in terms of the membrane shape and its stress tensor. We must outline that membrane-cable interface equilibrium leads to take into account a singular condition that makes the problem more difficult. Therefore, starting from the equilibrium equations, two dual problems can be considered namely direct problem and dual problem. Both problems will be defined and analyzed, studying their principal qualitative aspects. Particularly, for the direct problem a numerical resolution procedure is proposed, in order to obtain practical results.

Key-words: Membrane; Footbridge; Rigid boundary; Boundary cable; Direct problem; Dual problem.

\footnotetext{
(*) Universidad de Cádiz (UCA), (España)

${ }^{(* *)}$ Instituto de Ciencias de la Construcción Eduardo Torroja (CSIC), España 
1. Esquema de pasarela de membrana con cables de borde.

\section{INTRODUCCIÓN}

Este trabajo estudia el equilibrio bidimensional y continuo (superficie) de una membrana, en la fase de pretensado (esto es, cuando la misma se encuentra preparada para la fase de servicio), con bordes rígidos y cables de borde. Así, se considera el equilibrio de la membrana directamente para la forma que resulta al ser pretensada.

La idea de utilizar membranas en nuevas aplicaciones de ingeniería civil como las pasarelas implica, con relación a otros usos, una gran responsabilidad estructural y mayores esfuerzos, debidos a las cargas de uso, a la forma muy rebajada de la membrana y al fuerte pretensado preciso para alcanzar la rigidez requerida. En consecuencia, hace falta un adecuado análisis bidimensional. En este contexto, se está desarrollando en España una nueva tecnología de pasarelas (1).

El problema del pretensado está relacionado con los bordes de la membrana, elementos unidimensionales definidos por líneas en el espacio. Los cables de borde son curvos, definidos por curvas que pertenecen a la superficie de la membrana y, para mantener todo a tracción, su curvatura ha de estar orientada hacia el exterior de la misma, tal como muestra la Figura 1. Los bordes rígidos, capaces de trabajar a flexión, admiten cualquier forma. Sin embargo, tal como se verá, el equilibrio en la interfaz cable-membrana implica que los cables de borde no puedan adoptar cualquier forma.

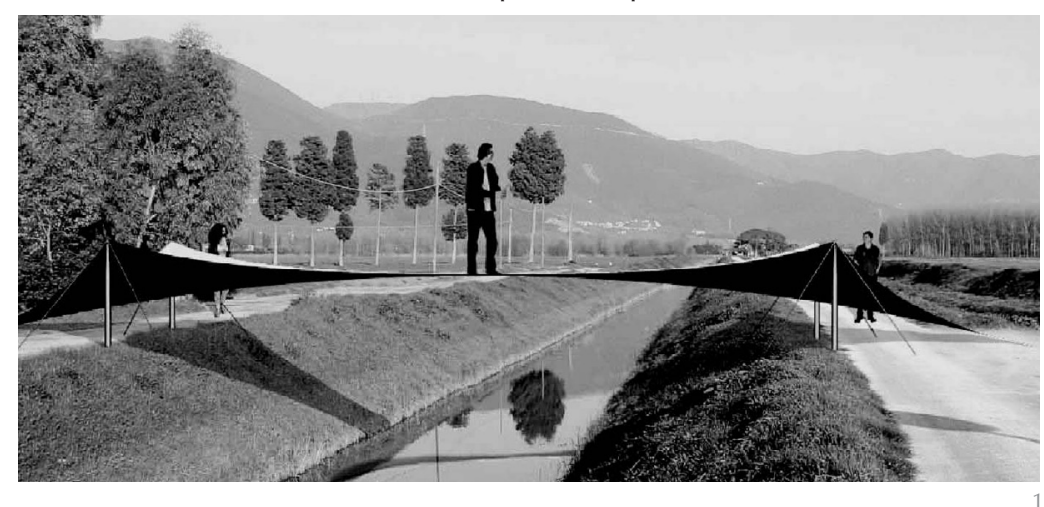

La consideración de bordes rígidos y cables de borde implica definir un problema más general que el analizado en el artículo (2). Como se verá, esto lleva a complicaciones importantes, tanto en términos de la formulación del problema como de su resolución.

Siguiendo la línea de dicho trabajo, los esfuerzos de la membrana se identifican con un tensor positivo de segundo orden y la forma con una superficie con curvatura de Gauss negativa. Así, es posible definir el equilibrio por medio de ecuaciones diferenciales en derivadas parciales, una de las cuales incluye los productos de variables asociadas a la forma de la membrana y al tensor de esfuerzos. Por tanto, si se fija el tensor de esfuerzos, puede considerarse el denominado problema directo $\mathrm{y}$, de modo complementario, el problema dual si lo que se fija es la forma de la membrana.

Con relación al equilibrio, si bien es cierto que las correspondientes ecuaciones en membranas son las mismas del análisis de los estados de membrana en láminas, ecuaciones bien conocidas (véanse, por ejemplo, la referencia (3)), también lo es que los problemas que se plantean son absolutamente diferentes, tal como se explica en el trabajo (2).

Como se ve en este trabajo, directamente del equilibrio por sí solo se pueden deducir bastantes cosas y, en particular, la forma de la membrana (cuando se fijan los esfuerzos), sin tener que considerar deformación alguna. Lo mismo sucede en el Método de la Densidad de Fuerzas, unidimensional (red superficial de cables en el espacio), basado exclusivamente en ecuaciones de equilibrio (4).

Una vez establecidas las ecuaciones de equilibrio interno para la membrana, es necesario definir las condiciones de frontera. Como se comentó antes, este trabajo es la continuación natural del artículo (2), porque generaliza el mismo problema considerando cables de borde además de bordes rígidos. Además, los cables de borde llevan a una formulación matemática de los problemas directo y dual que se complica sensiblemente con respecto al caso de bordes rígidos (véase, una vez más, la referencia (2)). Esto tiene mucho que ver con el hecho de que, precisamente, cable (1D) y membrana (2D) son estructuras singulares que no tienen rigidez propia (se la confiere la tracción).

Finalmente, una vez definidos rigurosamente los dos problemas, se estudian sus respectivos análisis cualitativos. Además, para el problema directo se presenta un procedimiento numérico capaz de proporcionar una buena estrategia de resolución. Este método se aplica a un caso real, la pasarela construida como prototipo (1), que se toma como referencia para evaluar los resultados. Por otro lado, se hacen algunas consideraciones sobre los aspectos matemáticos del problema dual.

\section{ECUACIONES DE EQUILIBRIO EN LA MEMBRANA}

Identifiquemos la membrana con una superficie $S$ con curvatura de Gauss negativa. En particular, $S$ es la gráfica de una función $z(x, y)$, definida en un dominio acotado $D$ del plano $x-y$, tal que $Z_{, x y} Z_{, y y}-Z^{2}{ }_{, x y}<0$ 
(la Figura 2 idealiza un elemento diferencial de membrana $d S$ y su correspondiente proyección $d D$ en el plano $x-y$, así como los relativos esfuerzos de membrana y proyectados, respectivamente $\tilde{N}_{\alpha \beta}$ y $N_{\alpha \beta}$, con $\left.\alpha, \beta=1,2\right)$

Como se sabe, el peso de la membrana es muy bajo y, en general, puede despreciarse para la fase de pretensado; más en particular para altos esfuerzos de pretensado como es el caso. Entonces, en esta fase no se considerará ninguna carga así que las ecuaciones de equilibrio serán homogéneas. Este aspecto no modifica la naturaleza del problema y es muy interesante desde el punto de vista del diseño (véase (2) para los detalles).

De esta forma, si $\sigma=N_{\alpha \beta}(\alpha, \beta=1,2)$ indica el tensor de esfuerzos proyectados, definido en $D$ (esto es, fuerza por unidad de longitud), las ecuaciones de equilibrio son [1]:

$N_{x x, x}+N_{x y, y}=0$ equilibrio en la dirección $x$, $\left\{N_{x y, x}+N_{y y, y}=0\right.$ equilibrio en la dirección $y$, $N_{x x} z_{x x}+2 N_{x y} z_{x y}+N_{y y} z_{y y}=0$ equilibrio en la dirección $z$,

Finalmente, la utilización de la función de Airy $H$, tal que [2]:

$H_{, x x}=N_{y y} H_{, x y}=-N_{x y} H_{, y y}=N_{x x^{\prime}}$

permite reducir el sistema [1] a la ecuación:

$H_{, x x} z_{, y y}-2 H_{, x y} z_{x y}+H_{, y y} z_{, x x}=0$

De esta forma, si se fija un tensor positivo $\sigma$ la función $z$ tiene que resolver una ecuación elíptica. Al contrario, una vez que se dé una superficie con curvatura de Gauss negativa, la función $H$ ha de resolver una ecuación hiperbólica (5).

Se pueden así definir dos problemas complementarios, el problema directo (se fijan los esfuerzos) y el problema dual (se fija la forma de la membrana).

\section{ECUACIONES DE EQUILIBRIO EN EL BORDE}

Con el objetivo de definir apropiadamente las condiciones de frontera para el problema directo y el problema dual, analicemos las correspondientes ecuaciones de equilibrio de borde.

Como ya se dijo, en este trabajo se analizarán ambos tipos de borde, rígido y cable; más en particular, en esta sección nos dedicaremos a estudiar las diferencias estructurales que los definen, concluyendo que la forma de un cable depende estrictamente de la forma de la membrana, mientras que el borde rígido puede asumir cualquier forma.

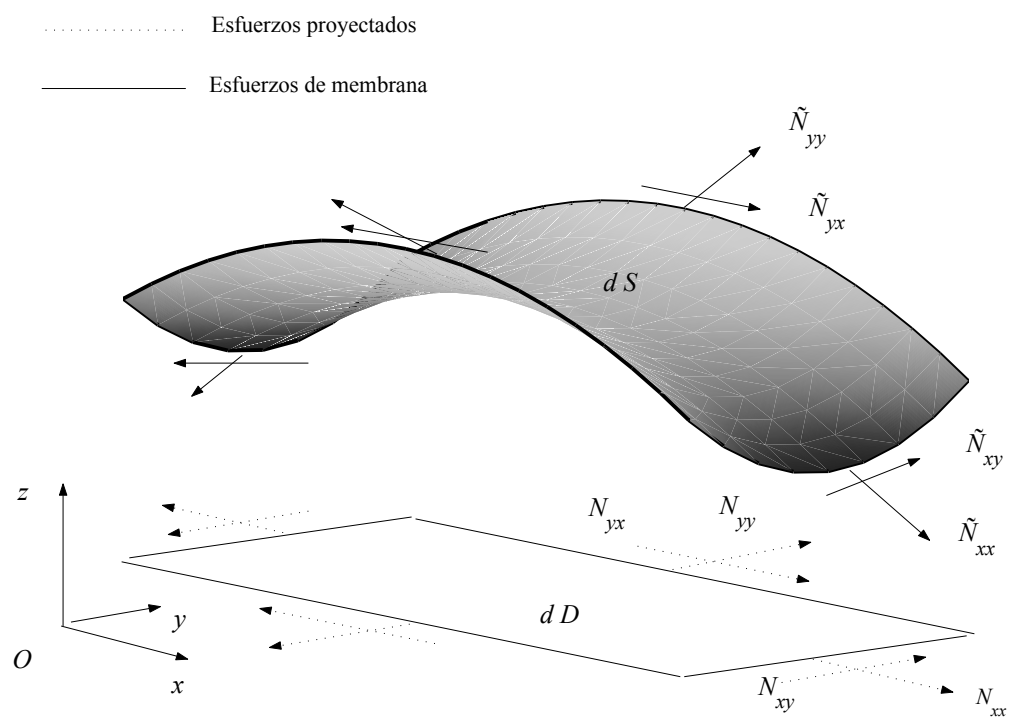

Identifiquemos el borde de la membrana con una curva espacial $C$; si $\tilde{N}_{\alpha \beta}(\alpha=1,2$ y $\beta=1,2)$ indica el tensor de esfuerzos sobre la membrana $S$ y $\tilde{f}=\left(\tilde{f}_{1}, \tilde{f}_{2}, \tilde{f}_{3}\right)$ la fuerza de borde repartida sobre $C$ (esto es, fuerza por unidad de longitud), resulta, al imponer el equilibrio, que $f$ es ortogonal al vector normal $N$ de la superficie $S$ a lo largo del contorno (para los detalles, véase la tesis (6)).

Además, siempre en (6), se comprueba que las ecuaciones de equilibrio para el borde tridimensional $C$ se reducen en las correspondientes para el borde plano $\Gamma=\partial D$, proyección horizontal de $C$. En este sentido, si $f=\left(f_{1}, f_{2}\right)$ indica la fuerza proyectada a lo largo de $\Gamma$ (fuerza por unidad de longitud), $\sigma=N_{\alpha \beta}$ el tensor de los esfuerzos proyectados y $n=\left(n_{1}, n_{2}\right)$ el vector normal (unitario) externo a $\Gamma$, el equilibrio de borde puede escribe como

$N_{\alpha \beta} n_{\beta}=f_{\alpha}(\operatorname{con} \alpha=1$ y 2$)$, sobre $\Gamma$

\subsection{Ecuaciones de equilibrio para el borde rígido}

En el caso en que se consideren sólo bordes rígidos, el equilibrio se reduce simplemente al sistema [4], tal y como se analiza en (2).

Por ejemplo, si la forma del borde rígido $\Gamma^{r}$ en el plano horizontal $x$ - $y$ está definido por rectas verticales, así como se hará más adelante para propósitos prácticos (véase la Figura 4), el sistema [4] se escribe como:

$\left\{\begin{array}{l}N_{x x}=f_{1} \text { sobre } \Gamma^{r} \\ N_{y y}=f_{2} \text { sobre } \Gamma^{r}\end{array}\right.$

De esta forma, el mismo sistema [4] permite obtener $f$ una vez que se fije $N_{\alpha \beta}$ y viceversa. 


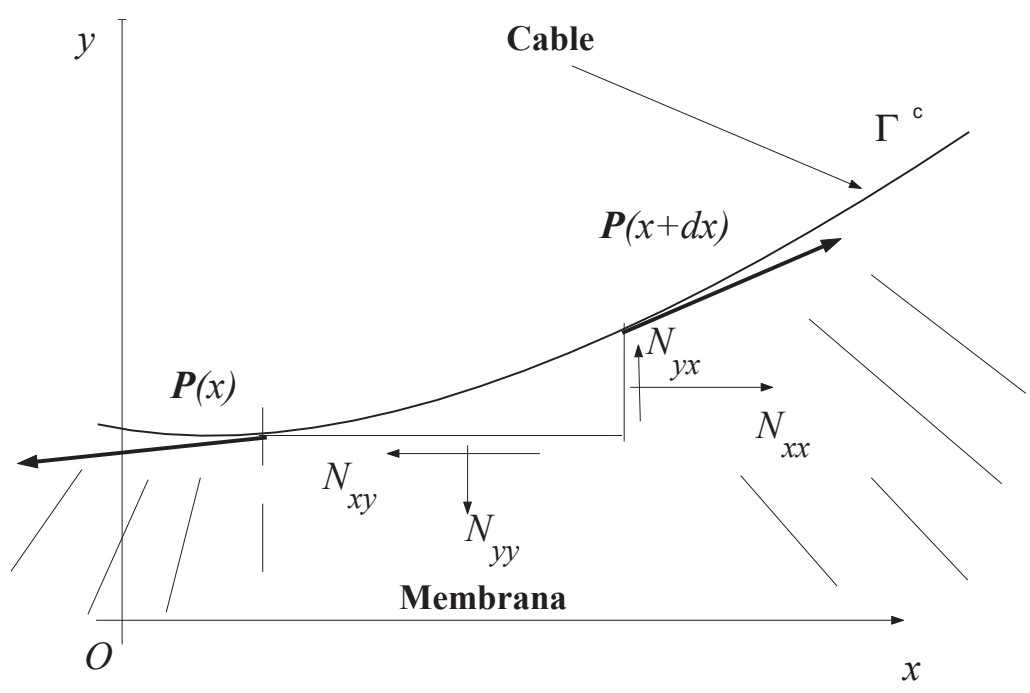

3. Interfaz cable-membrana ( $P$ representa la proyección de $\tilde{P}$ ).

\subsection{Ecuaciones de equilibrio para el cable de borde}

Si parte del contorno de la membrana está compuesto por un cable de borde, en el sistema [4] la fuerza correspondiente $f$ se expresa tanto en términos de la tensión como de la forma del mismo cable.

Identifiquemos el cable con una curva $C=(x, y(x), h(x))$ siendo $h(x)=z(x, y(x))$ la forma espacial del mismo cable,obtenida "levantando" el cable proyectado $y(x)$ por medio de la membrana $z(x, y)$. La tensión del cable es el vector tangente expresado por

$$
\tilde{P}=\frac{\tilde{P}}{\sqrt{1+y^{\prime}+h^{\prime}}}\left(1, y^{\prime}, h^{\prime}\right),
$$

donde $\tilde{P}$ representa el valor de la tensión. De esta forma, la componente $x$ de $\tilde{P}$ es

$$
P_{x}=\frac{\tilde{P}}{\sqrt{1+y^{1^{2}+h^{\prime}}}},
$$

que utilizaremos a continuación. Con referencia a la Figura 3, es posible comprobar que el sistema [5]:

$\left\{P_{x}^{\prime}=N_{x y}-y^{\prime} N_{x x}\right.$,

$\left\{\begin{array}{l}x \\ \left(P_{x} y^{\prime}\right)^{\prime}=N_{y y}-y^{\prime} N_{x y},\end{array}\right.$

representa el equilibrio plano cable-membrana; este último sistema es un caso particular del sistema [4].

Por lo que se refiere al equilibrio en la dirección $z$, al tener en cuenta el sistema [5], se puede verificar que el equilibrio vertical cable-membrana se simplifica en la ecuación

$\left(P_{x} h^{\prime}\right)^{\prime}=-z_{, x} y^{\prime} N_{x x}+z_{, x} N_{x y}-y^{\prime} N_{x y} z_{, y}+z_{, y} N_{y y}$
Además, es posible comprobar que la última ecuación se expresa como:

$\left(P_{x} h^{\prime}\right)^{\prime}=P_{x}^{\prime} z_{, x}+\left(y^{\prime} P_{x}\right)^{\prime} z_{, y}$

Por otra parte, por medio de un simple cálculo (véase (6), para los detalles), se podría argumentar que la ecuación [6], considerando una vez más también el sistema [5], es equivalente a

$z_{, x x}+2 y^{\prime} z_{, x y}+y^{\prime 2} z_{, y y}=0$

Esta última relación, que representa la ecuación de compatibilidad entre el cable y la membrana, implica que la fuerza repartida $\tilde{f}$ no sólo es ortogonal a la normal $\mathrm{N}$ a la superficie $S$ sino que pertenece al plano osculador de la curva $C$.

Para comprobarlo, si $N$ es el vector unitario normal a $S$, y $t$ y $n$ aquellos tangente y normal al cable $C$, es suficiente imponer que los vectores $t$ y $n$ son ortogonales a $N$. Siendo $t$ proporcional a $\left(1, y^{\prime}, z_{, x}+z_{, y} y^{\prime}\right), n$ a $\left(0, y^{\prime \prime}, z_{, x x}+\right.$ $\left.2 y^{\prime} z_{, x y}+y^{\prime} z_{, y y}+y^{\prime \prime} z_{, y}\right)$ y $N$ a $\left(z_{, x} z_{, y},-1\right)$, se debe de cumplir $z_{, x x}+2 y^{\prime} z_{, x y}+y^{\prime} z_{, y y}=0$

En este trabajo, al tenerse en cuenta los cables de borde, se utilizará el siguiente sistema para expresar el equilibrio cable-membrana [8]:

$\left\{\begin{array}{l}\left.P_{x}^{\prime}=N_{x y}-y^{\prime} N_{x x} \text { (equilibrio en la dirección } x\right) \\ \left.\left(P_{x} y^{\prime}\right)=N_{y y}-y^{\prime} N_{x y} \text { (equilibrio en la dirección } y\right) \\ \left.\left(P_{x} h^{\prime}\right)=P^{\prime} z_{x}+\left(y^{\prime} P_{x}\right)^{\prime} z_{y} \text { (equilibrio en la dirección } z\right)\end{array}\right.$

Al revés, la tesis referenciada en [8] analiza el mismo problema partiendo del sistema anterior en el que se sustituye su última ecuación por la expresión [7].

\section{EL PROBLEMA DIRECTO: DEFINICIÓN Y PROPIEDADES}

En esta sección definiremos y analizaremos el problema directo, proponiendo, además, un procedimiento numérico para el cálculo aproximado de su solución.

\section{Problema directo}

Con referencia al equilibrio expresado por el sistema [1], sea $N_{\alpha \beta}$ un tensor positivo $y$ simétrico del segundo orden tal que

$\left\{\begin{array}{l}N_{x x, x}+N_{x y, y}=0 \\ N_{x y, x}+N_{y y, y}=0\end{array} \leftrightarrow \sum_{\beta=1}^{2} N_{\alpha \beta, \beta}=0\right.$

$\alpha=1,2$ en un domino acotado $D$ del plano $x-y$.

Hallar la superficie $z$, definida en $D$, de manera que 
$\left\{\begin{array}{l}z_{x x} N_{x x}+2 z_{x y} N_{x y}+z_{, y y} N_{y y}=0 \text { en } D, \\ z=g \text { sobre } \Gamma^{r} \\ \left(P_{x} h^{\prime}\right)^{\prime}=P_{x}^{\prime} z_{, x}+\left(y^{\prime} P_{x}\right)^{\prime} z_{, y} \text { sobre } \Gamma^{c}\end{array}\right.$

siendo $h(x)=z(x, y(x))$ la forma espacial del cable, $\Gamma^{r}$ la parte del contorno de $D$ compuesto por bordes rígidos, $\Gamma^{c}$ la correspondiente a los cables de borde, y $g(y)$ el valor de $z$ a lo largo del mismo borde rígido $\Gamma^{r}$.

Debido a la segunda condición de frontera, el anterior no es un típico problema elíptico con condición usual de Dirichlet o DirichletNeumann (véanse $(7,8)$ ). En la tesis $(6)$, se comprueba por medio de rigurosos resultados matemáticos la unicidad de la solución. En el siguiente apartado, se propondrá un método numérico útil para el cálculo aproximado de la solución; no obstante, hay que matizar que argumentar la existencia del mismo sistema [9] no parece ser del todo inmediato.

Por supuesto, si el borde de la membrana es totalmente rígido (esto es $\Gamma^{c}=\varphi$ ), el sistema [9] se reduce a un problema de Dirichlet, ya profundizado en el trabajo (2).

Antes de desarrollar el método de resolución del problema directo, es oportuno matizar algunos aspectos relativos al sistema [8]. En particular, tal y como manifiestan las ecuaciones de equilibrio que lo definen, es posible calcular, a partir de los esfuerzos $N_{\alpha \beta}$ la forma del cable de borde proyectado $y(x)$ que, juntamente con el borde rígido $\Gamma^{r}$ permite fijar el dominio $D$ (véase Figura 4). De la misma forma, es posible calcular $P_{x^{\prime}}$ componente de la tensión del cable en la dirección $x$, a partir de un cierto valor $P_{x}^{0}$ conocido. No obstante, la forma espacial del cable (expresada por la función $h(x)$ es una incógnita del problema, que se obtiene al conocer la forma de la membrana $z(x, y)$.

\subsection{Método de resolución para el problema directo}

Las condiciones $\sum_{\beta=1}^{2} N_{\alpha \beta, \beta}=0,(\alpha=1,2)$ permiten escribir el sistema [9] en la siguiente forma divergencial [10]:

$$
\left\{\begin{array}{l}
\operatorname{div}(\sigma \cdot \nabla z)=0 \text { en } D \\
z=g \text { sobre } \Gamma^{r} \\
\left(P_{x} h^{\prime}\right)^{\prime}=P_{x}^{\prime} z_{, x}+\left(y^{\prime} P_{x}\right)^{\prime} z_{, y} \text { sobre } \Gamma^{c}
\end{array}\right.
$$

Este último sistema será el punto de partida para el análisis del problema directo.

A continuación, utilizando el Método de los Elementos Finitos (véanse, por ejemplo, $(9,10)$, se propondrá una técnica capaz de proporcionar la solución aproximada del sistema [10].

Reescribamos el problema [10], considerando por un lado el sistema reducido (con condición de frontera clásica, de tipo Dirichlet) [11]:

$\left\{\begin{array}{l}\operatorname{div}(\sigma \cdot \nabla z)=O \text { en } D \\ z=g \text { sobre } \Gamma^{r} \\ z=h \text { sobre } \Gamma^{c}\end{array}\right.$

y, por otro, la ecuación [12]:

$\left(P_{x} h^{\prime}\right)^{\prime}=P_{x}^{\prime} z_{x}+\left(y^{\prime} P_{x}\right)^{\prime} z_{, y}$ sobre $\Gamma^{c}$

Una vez fijada una malla para $D$, indiquemos con $n_{t}$ el número total de nodos en $D, n_{r}$ el número total de nodos sobre $\Gamma^{r}$ y $n_{c}$ el número total de nodos sobre $\Gamma^{c}$. Si con $N_{j}$ nos referimos a las funciones de forma lineales, reemplazando las correspondientes aproximaciones $z \cong \sum_{j=1}^{n_{t}} z_{j} N_{j}$ y $h^{\sim \sum_{j=1}^{n_{c}}} h_{j} N_{j}$ en el sistema [11], se obtiene el siguiente sistema lineal[13].

$\left(\begin{array}{ccc}K & A_{r}^{T} & A_{c}^{T} \\ A_{r}^{T} & 0 & 0 \\ A_{c}^{T} & 0 & 0\end{array}\right)\left(\begin{array}{l}z \\ \lambda_{r} \\ \lambda_{c}\end{array}\right)=\left(\begin{array}{l}0 \\ g \\ h\end{array}\right)$

En la última ecuación matricial, $z=\left(z_{1}, \ldots, z_{t}\right)$ $\boldsymbol{g}=\left(g_{1}, \ldots, g_{r}\right)$ y $\boldsymbol{h}=\left(\boldsymbol{h}_{1}, \ldots, \boldsymbol{h}_{\mathrm{c}}\right)$ indican el vector nodal de $z$ en $D$, de $g$ sobre $\Gamma^{c}$ de $h$ sobre $\Gamma^{c}$, $k$ la matriz de rigidez, $A_{\gamma}$ y $A_{C}$ las matrices de los Multiplicadores de Lagrange, y $\lambda_{\gamma}$ y $\lambda_{c}$ los vectores de los Multiplicadores de Lagrange.

Sea, ahora, z la solución del sistema [13]: puede escribirse

$z=\mathcal{H} h+\mathcal{G g}$

siendo

$\mathcal{H} \in M_{\mathrm{n}_{\nu} \mathrm{n}_{c}}(\mathcal{R})$ y $\mathcal{G} \in M_{\mathrm{n}_{\nu} \mathrm{n}_{c}}(\mathcal{R})$

Además, teniendo en cuenta la ecuación [12], puede considerarse la ecuación $n_{c}$ - dimensional, definida por

$$
\begin{array}{r}
\int_{\Gamma^{c}}\left[P^{\prime}{ }_{x} Z_{, x}+\left(y^{\prime} P_{x}\right)^{\prime} z_{, y}-\left(P_{x} h^{\prime}\right)^{\prime}\right] N_{i} d \Gamma^{c}=0 \\
i=1,2,3 \ldots, n_{c}
\end{array}
$$

Reemplazando, igual que antes, las aproximaciones $z \cong \sum_{j=1}^{n_{t}} z_{j} N_{j}$ y $h \cong \sum_{j=1}^{n_{i}} h_{j} N_{j}$ e integrando por partes, se obtiene la expresión:

$$
\sum_{j=1}^{n_{t}} z_{j} \int_{\Gamma^{c}}\left[N_{j, x} P^{\prime}{ }_{x}+N_{j, y}\left(y^{\prime} P_{x}\right)^{\prime}\right] N_{i} d \Gamma^{c}+\sum_{j=1}^{n_{c}} h_{j} \int_{\Gamma^{c}} P_{x} N^{\prime}{ }_{j} N^{\prime}{ }_{i} d \Gamma^{c}=0,
$$

Finalmente, definiendo,

$$
i=1,2,3 \ldots, n_{c}
$$

$$
\begin{aligned}
& M_{i j}=\int_{\Gamma^{c}}\left[N_{j, x} P^{\prime}{ }_{x}+N_{j, y}\left(y^{\prime} P_{x}\right)^{\prime}\right] N_{i} d \Gamma^{c} \\
& \text { y } W_{i j}=\int_{\Gamma} P_{x} N^{\prime}{ }_{j} N^{\prime}{ }_{i} d \Gamma^{\prime}
\end{aligned}
$$


se conviene al siguiente sistema

$\mathcal{M} z+\mathcal{W h}=0$

en el que

$\mathcal{M} \in M_{\mathrm{n}_{\sigma} \mathrm{n}_{\mathrm{t}}}(\mathbb{R})$ y $\mathcal{W} \in M_{\mathrm{n}_{c} \mathrm{n}_{c}}(\mathbb{R})$

Comparando los sistemas [14] y [16], se obtiene la siguiente expresión para la solución:

$h=(\mathcal{M H}+\mathcal{W})^{-1} \mathcal{M G g}$

siendo $h$ el vector nodal de $z$ a lo largo de $\Gamma^{\mathrm{c}}$, que nos proporciona una aproximación tridimensional de la forma del cable.

De la misma manera, es posible obtener la solución $z$,

$z=\mathcal{H} h+\mathcal{G g}$

siendo, ahora, $h$ el vector nodal de $z$ en $D$, que nos proporciona una aproximación tridimensional de la forma de la membrana.

\subsection{Un ejemplo concreto de problema directo}

Apliquemos el procedimiento numérico previamente analizado para resolver el caso de la pasarela peatonal construida en España. Es importante comentar que el caso presentado en el ejemplo que sigue es casi igual, pero no exactamente, al llevado a cabo en la realidad. Ello es debido a que, recordando que se trata de encontrar la forma de la membrana (y de los cables de borde), el método de cálculo para ello en el caso real fue aproximado. Así, las formas por una y otra vía no pueden ser idénticas y, en consecuencia, ambos casos tampoco pueden serlo, solamente bastante aproximados.

En efecto, en el caso real, se realizó una aproximación analítica, ajustando expresiones para los esfuerzos y las formas de membrana (superficie) y cables de borde a todas las ecuaciones de equilibrio ya vistas; cuya solución, aproximada pero bastante precisa, implicaba valores de los esfuerzos de membrana en las direcciones de los ejes casi constantes pero no exactamente véase (1).
Por su parte, aquí se aplica el citado procedimiento y, como de todos modos no puede obtenerse un caso idéntico, se simplifica fijando esfuerzos de membrana constantes, muy aproximados a los anteriores (valores diferentes en las direcciones de los ejes).

\section{Datos para el ejemplo}

Todas las longitudes ( $x, y, z$, así como las longitudes en las figuras) se expresan en metros.

El dominio $D$ tiene la forma mostrada en la Figura 4:

$D:=\left\{(x, y) \in \mathbb{R}^{2}\right.$ tal que $\left.-a \leq x \leq a y-y(x) \leq y \leq y(x)\right\}$ siendo $a=5 \mathrm{~m}$ e $y=x$ obtenida resolviendo el sistema [5].

Las condiciones sobre el borde rígido y el tensor de esfuerzos son:

$\left\{\begin{array}{l}g(y)=\frac{17}{800} y^{2}\left(11-\frac{1}{6} y^{2}\right) \text { forma de } z \text { a lo largo de } \Gamma^{\prime} \\ N_{x x}=10 \mathrm{kN} / \mathrm{m}, N_{x y}=0 \mathrm{kN} / \mathrm{m}, N_{y y}=4 \mathrm{kN} / \mathrm{m} .\end{array}\right.$

De esta forma, el sistema [5] se resume en $\left\{\begin{array}{l}P_{x}^{\prime}=-10 y^{\prime} \\ \left(P_{x} y^{\prime}\right)^{\prime}=4\end{array}\right.$

$\varepsilon_{+}: \frac{8}{605} x^{2}+\frac{4}{121}\left(y-\frac{13}{2}\right)^{2}=1$ y $\varepsilon_{-}: \frac{8}{605} x^{2}+\frac{4}{121}\left(y+\frac{13}{2}\right)^{2}=1$.

Integrando, usando la simetría e imponiendo $y( \pm 5)=2$ y $y(0)=1$ y $y( \pm 5)=-2$ y $y(0)=1$, se obtienen las dos elipses, $\varepsilon_{+}$y $\varepsilon_{\text {. }}$

Escogiendo las ramas adecuadas de $\varepsilon_{+}$y $\varepsilon_{\text {. }}$ (gráfica (a) de la Figura 5), es natural fijar e

$D:=\left\{(x, y) \in \mathbb{R}^{2}\right.$ tal que $\left.-a \leq x \leq a y-y(x) \leq y \leq y(x)\right\}$

$y(x)=\frac{13}{2}-\sqrt{\frac{121}{4}-\frac{2}{5} x^{2}}$

(la gráfica (b) de la Figura 5 representa una malla compuesta por 477 nodos).

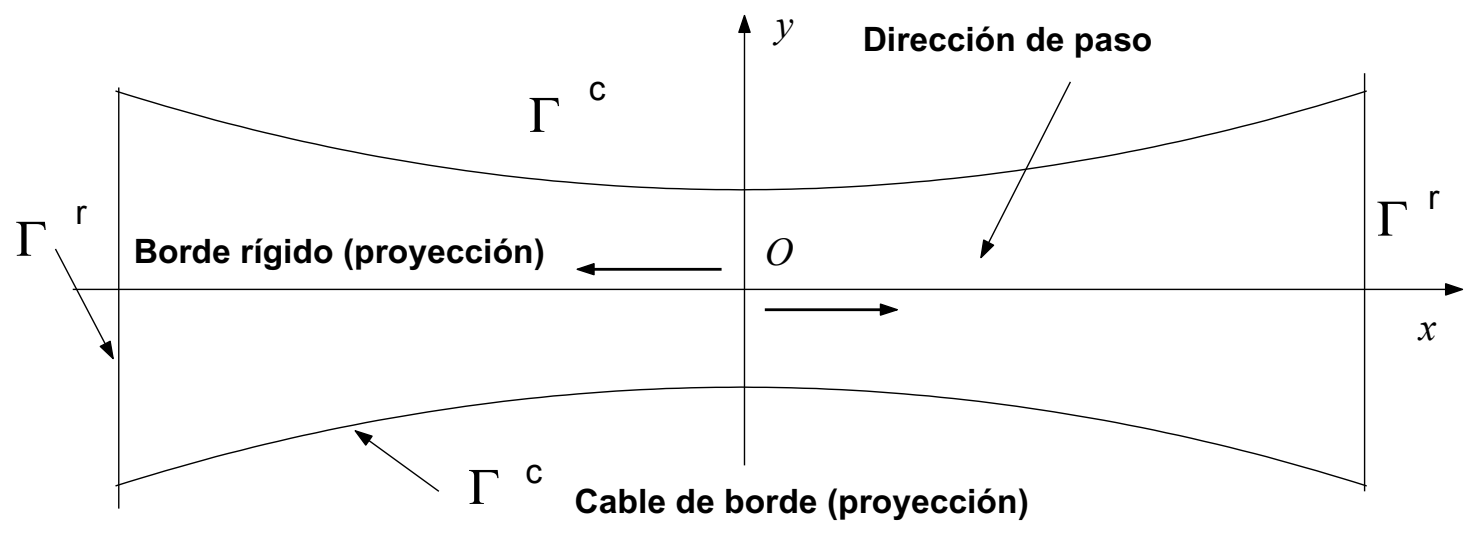


En estas condiciones, hay que resolver el sistema

$$
\begin{aligned}
& 10 z_{, x x}+4 z_{, y y}=0 \text { en } D, \\
& z=\frac{17}{800} y^{2}\left(11-\frac{1}{6} y^{2}\right) \text { sobre } x=\mp 5\left(\text { i.e } \Gamma^{\mathrm{r}}\right), \\
& z=h \text { sobre } y(x)=\mp\left(\frac{13}{2}-\sqrt{\frac{121}{4}-\frac{2}{5} x^{2}}\right)\left(\text { i.e. } \Gamma^{\mathrm{c}}\right), \\
& \left(P_{x} h^{\prime}\right)^{\prime}=P^{\prime}{ }_{x} z_{, x}+\left(y^{\prime} P_{x}\right)^{\prime} z_{, y} \quad \text { sobre } y(x)=\mp\left(\frac{13}{2}-\sqrt{\frac{121}{4}-\frac{2}{5} x^{2}}\right) \quad\left(\text { i.e. } \Gamma^{\mathrm{c}}\right)
\end{aligned}
$$

Método Iterativo del Punto Fijo $(7,8)$, ajustando "paso a paso" una cierta forma inicial $h_{0}$, de manera que en el límite se satisfagan todas las ecuaciones del mismo sistema [9].
5. Dominio D escogido para el ejemplo del problema directo.

Sub-figura (a): Cables de borde (ramas de elipse).

Sub-figura (b): Malla de 477 nodos para el dominio $\mathrm{D}$.
Las gráficas (a) de las Figuras 6 y 7 representan los resultados numéricos obtenidos, tomando como punto más bajo de la membrana (esto es, el extremo $(-5,0)$ el origen de las medidas. Estos resultados han sido calculados ejecutando un programa en MATLAB, basado en la utilización de elementos cuadriláteros lineales (es posible encontrar los detalles del método en la tesis (6).

Como ya se ha comentado anteriormente, el caso ahora analizado no se corresponde a aquél real estudiado en el trabajo (1). A pesar de ello, comparando sendas soluciones obtenidas, es posible confirmar que las mismas son del todo coherentes. De hecho, por ejemplo, la flecha del cable calculada en el caso real es de 0,31 $\mathrm{m}$, contra el valor de $0,30 \mathrm{~m}$ obtenido por medio del método numérico aquí propuesto.

Aparte ello, también es posible analizar la componente de la tensión del cable $P_{x}$.

En fecto, debido a la relación

$P^{\prime}{ }_{x}=-10 y^{\prime}$

imponiendo por ejemplo

$P_{x}^{0}=P_{x}(0)=55 k N$, se obtiene la expresión

$P_{x}=10 \sqrt{\frac{121}{4}-\frac{2}{5} x^{2}}$

Finalmente, cabe subrayar que el procedimiento utilizado presenta una cierta inestabilidad numérica. De hecho, la gráfica (a) de la Figura 6 muestra que la solución numérica para el cable $h=h(x)$ varía en términos del número de nodos utilizados; en particular, no es posible observar un comportamiento estrictamente monótono para la solución. Esto parece estar relacionado con la formulación del problema y no tanto con la aproximación de elementos finitos empleados. En efecto, analizando en detalle las ecuaciones de equilibrio, podemos notar que en el sistema [9] se fuerza a la incógnita $z$ a que verifique en el borde una ecuación del mismo orden que la que ha de cumplirse en el interior del dominio, siendo lo usual que en la frontera la incógnita cumpla condiciones de órdenes inferiores.

Una posible alternativa para la resolución, sobre la que los autores están trabajando, se basa en buscar la forma final del cable $h$ mediante el

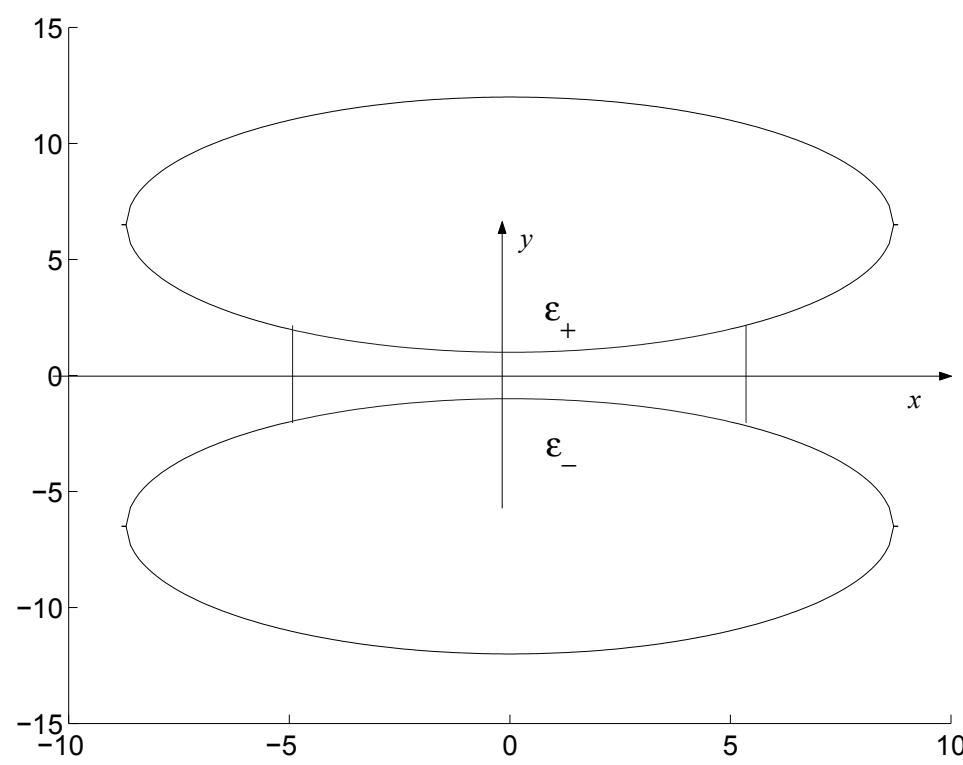

5 a

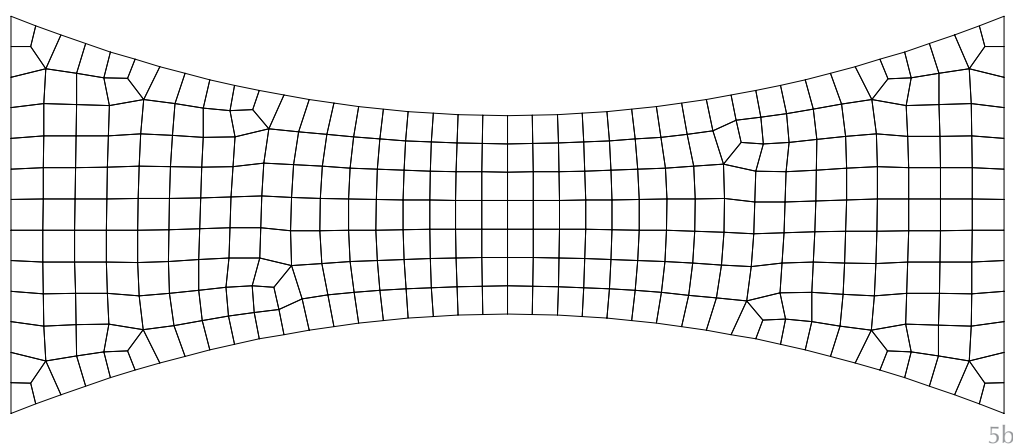

\section{PROBLEMA DUAL: DEFINICIÓN Y PROPIEDADES}

En este apartado se definirá y analizará el problema dual.

\section{Problema dual}

Con referencia al equilibrio expresado por el sistema [1], sea $S=(x, y, z(x, y))$ una superficie con curvatura de Gauss negativa. Al resolver la ecuación [7] se obtiene una función $y(x)$ que define el cable de borde proyectado, indicado con $\Gamma^{c}$.

Si con $f=f(y)$ se denota la fuerza sobre el borde rígido $\Gamma^{r}$, hallar el tensor de esfuerzos $\sigma=N_{\alpha \beta}$ en el dominio $D$, tal que 

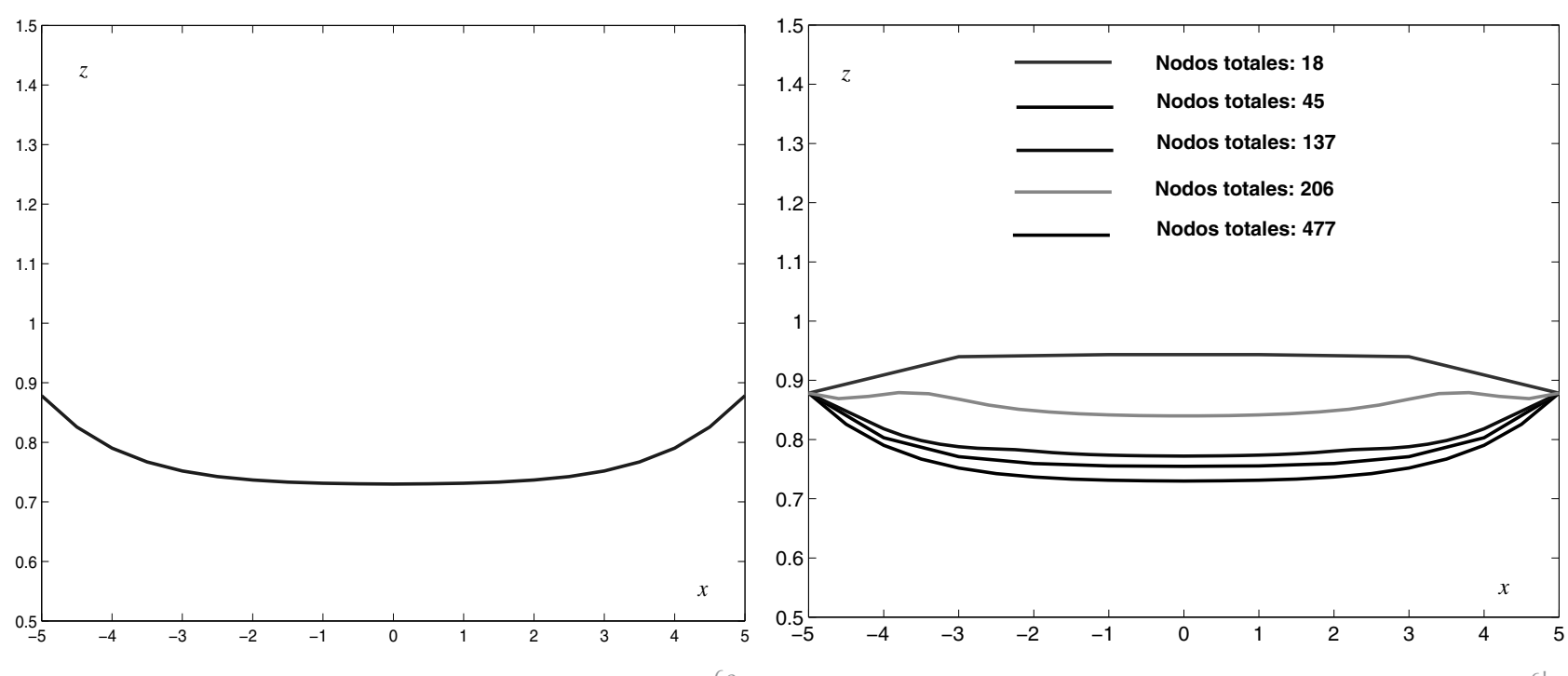

$6 a$

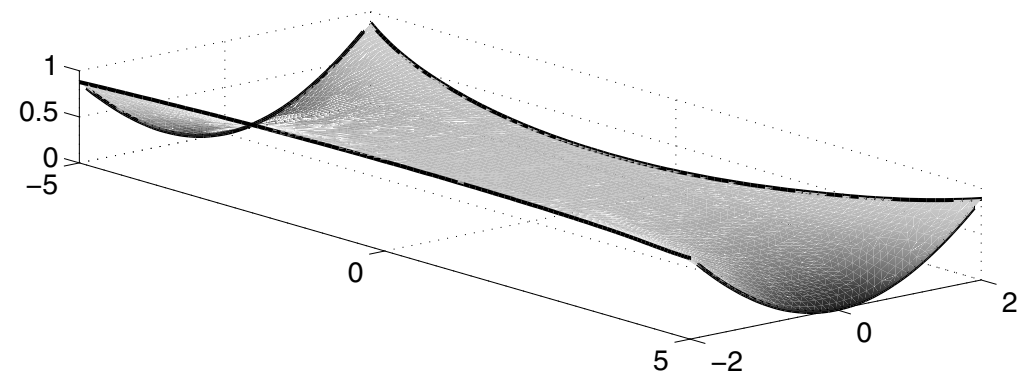

m

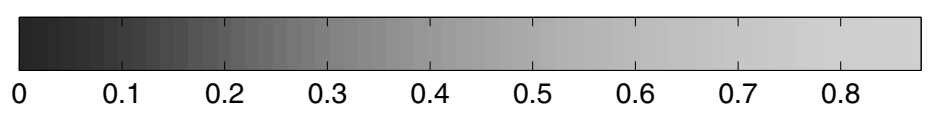

6a. Solución numérica calculada usando 137 nodos; proyección vertical del cable.

6b. Solución numérica de $h=h(x)$; proyección vertical del cable en términos del número de nodos.

7. Pasarela real y gráfica de su forma calculada numéricamente

Sub-figura (a): Solución numérica $z=z(x, y)$.

$$
y^{\prime}(x)=\frac{-z_{, x y} \pm \sqrt{z_{, x y}^{2}-z_{, x x} z_{, y y}}}{z_{, y y}},
$$

que se reduce a

$z_{, x x}+2 y^{\prime} z_{, x y}+y^{\prime 2} z_{, y y}=0$

Como ya se comentó, esta relación, junta con las del sistema [5], es equivalente a

$\left(P_{x} h^{\prime}\right)^{\prime}=P_{x}^{\prime} z_{, x}+\left(y^{\prime} P_{x}\right)^{\prime} z_{, y}$

Todo ello implica que en el problema dual las curvas que representan los cables de borde coinciden con las curvas características de la ecuación. En este sentido, deberíamos considerar un problema hiperbólico con condiciones de frontera definidas a lo largo de sus curvas características. Tal y como se detalla en (5), no es siempre posible garantizar la existencia, unicidad y estabilidad de la solución para este tipo de problemas.

Por tanto, recordando también los casos de bordes rígidos (2), en el problema dual las ecuaciones de equilibrio pueden no ser suficientes para calcular la distribución de esfuerzos que equilibra la forma previamente fijada; al revés de lo que ocurre en el problema directo. En otras palabras, el problema dual no es siempre isostático. Ahora bien, desde el punto de vista estructural, está claro que si se consideraran también las ecuaciones constitutivas y de compatibilidad no nos enfrentaríamos a este tipo de singularidad, obteniendo un problema hiperbólico coherente y bien planteado.

Además, al analizar las curvas características de la ecuación de equilibrio de membrana

$H_{, x x} Z_{, y y}-2 H_{, x y} z_{, x y}+H_{, y y} Z_{, x x}=0$

se obtiene la ecuación diferencial (ordinaria)
En fin, con relación al problema dual, conviene indicar que en (6) se ofrece una casuística de ejemplos analíticamente resolubles para borde rígido, a partir de algunos resultados propios de la ecuación de ondas. 


\section{RESUMEN Y CONCLUSIONES}

En este trabajo se ha analizado el equilibrio de una membrana, para la fase de pretensado, en el caso general en que sus bordes están compuestos por elementos rígidos y cables. Se han formulado las ecuaciones de equilibrio sobre la superficie y los bordes de la membrana mediante variables asociadas a la forma y a los esfuerzos de todos ellos. Así, según el tipo de variables fijadas, se definen dos problemas: el problema directo y el problema dual.

Este tipo de análisis riguroso, en el continuo bidimensional, está asociado a los fuertes requisitos estructurales de una nueva tecnología de pasarelas con estructura de membrana que está siendo desarrollada en España.

Un aspecto relevante del trabajo es el análisis bidimensional en los bordes, particularmente en el caso de cables, cuya forma, al contrario que los bordes rígidos, no puede fijarse arbitrariamente a priori.

Hay que destacar, en particular, que el equilibrio en la interfaz cable-membrana conduce a una ecuación singular, que implica considerar un del todo inusual y complejo problema diferencial de contorno. Esto se relaciona con el hecho de que ahí interaccionan precisamente las dos estructuras singulares sin rigidez propia, cable (1D) y membrana (2D), que no pueden adoptar cualquier forma para estar en equilibrio.
Más precisamente, si por un lado es cierto que el problema directo se basa en una ecuación diferencial elíptica, por otro la condición de contorno asociada no es ni de tipo Dirichlet ni de tipo Dirichlet-Neumann. En este sentido, aunque es posible argumentar de forma rigurosa la unicidad de la solución, no es inmediato demostrar su existencia. Sin embargo, desde un punto de vista práctico, puede proponerse un procedimiento numérico, como aquí se ha hecho empleando el Método de los Elementos Finitos, para la construcción de la solución.

Además, se ha resuelto un caso particular de problema directo mediante el método numérico y se han comparado sus resultados con un caso real de referencia. La comparación muestra valores similares y coherentes. Por otro lado, en el método aquí propuesto es posible observar una cierta inestabilidad, debida a la formulación intrínseca del problema.

Finalmente, el problema dual es de tipo hiperbólico, para el cual no siempre es posible proporcionar una solución, única y estable; esto es, en el problema dual no siempre existe isostatismo. Pero, además, cuando existen cables de borde, la condición de equilibrio entre cable y membrana implica que los cables de borde coinciden con las curvas características de la ecuación diferencial. Por tanto, el problema dual con condiciones de frontera definidas a lo largo de dichos cables no es en general matemáticamente admisible.

\section{BIBLIOGRAFÍA}

(1) Murcia J.: "Tecnología de pasarelas con estructura de membrana". Informes de la Construcción; vol. 59, n. ${ }^{\circ}$ 507, 21-31, 2007.

(2) Viglialoro G.; Murcia J.; Martínez F.: "Problemas asociados al equilibrio en estructuras de membrana con bordes rígidos". Informes de la Construcción; vol. 61, n. ${ }^{\circ}$ 516, 57-66, 2009.

(3) Timoshenko S.; Woinowsky-Krieger S.: Theory of Plates and Shells. McGraw-Hill, Inc., New York, 1959.

(4) Linkwitz K.: "About formfinding of double-curved structures". Engineering Structures; vol. 21, pp. 709-718, 1999.

(5) Hörmander L.: Linear Partial Differential Operators. Springer, Berlín, 1964.

(6) Viglialoro G.: Análisis matemático del equilibrio en estructuras de membrana con bordes rígidos y cables. Pasarelas: forma y pretensado. Tesis doctoral, Universidad Politécnica de Cataluña. ISBN: 978-84-690-6482-5, 2006. Depósito legal: B.33064-2007. Enlace: http://www.tesisenxarxa.net/TDX-0515107-100745/.

(7) Brezis H.: Análisis funcional: teoría y aplicaciones. Madrid, Alianza Editorial, 1983.

(8) Gilbarg L.; Trudinger N. S.: Elliptic Partial Diferential Equations of Second Order. Springer, 1998.

(9) Hughes T. J. R.: The Finite Element Method. Linear Static and Dynamic Finite Element Analysis. Mineola, New York Dover Publications, 1987.

(10) Zienkiewicz O. C.; Taylor R. L.: The Finite Element Method. Butterworth Heinemann, 2000. 